\title{
Quality of life of older Malaysians living alone.
}

\begin{abstract}
According to the 2000 census report, about $7 \%$ of the 1.4 million people 60 years and over in Malaysia live alone. This study investigated socioeconomic factors affecting the quality of life of this vulnerable population. Data from a subsample of the study on Mental Health and Quality of Life of Older Malaysians were used in this paper. About $10 \%$ of the original sample or 299 older persons aged 60 years and over were living alone. Perceived quality of life was measured by asking "In general, how do you perceive your quality of life?" on a 5point Likert Scale response. A multinomial regression model was utilized to identify variables associated with perceived quality of life in older person living alone. Results revealed four factors significantly predicted their perceived quality of life, which include self rated health, gender, employment status, and level of education. In conclusion, self rated health and level of education had significant positive effects on perceived quality of life; elderly women and employed older persons were more likely to fall in the poor perceived quality of life group. The findings of this study posited that self-rated health and level of education have significant positive effects on the perceived quality of life; being female and employed are related to lower quality of life of an older person living alone.
\end{abstract}

Keyword: Quality of life; Older Malaysians; Living alone. 